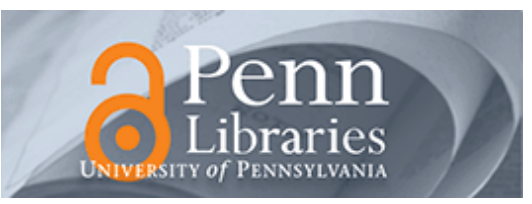

University of Pennsylvania

ScholarlyCommons

\title{
Self-organized cortical map formation by guiding connections
}

Stanley Y.M. Lam

HKUST

Bertram E. Shi

HKUST

Kwabena A. Boahen

University of Pennsylvania, boahen@seas.upenn.edu

Follow this and additional works at: https://repository.upenn.edu/be_papers

\section{Recommended Citation}

Lam, S. Y., Shi, B. E., \& Boahen, K. A. (2005). Self-organized cortical map formation by guiding connections. Retrieved from https://repository.upenn.edu/be_papers/136

Copyright IEEE 2005. Reprinted from:

Lam, S.Y.M.; Shi, B.E.; Boahen, K.A., "Self-organized cortical map formation by guiding connections," Circuits and Systems, 2005. ISCAS 2005. IEEE International Symposium on, vol., no., pp. 5230-5233 Vol. 5, 23-26 May 2005 URL: http://proxy.library.upenn.edu:2994/stamp/stamp.jsp?arnumber=1465814\&isnumber=31469

This material is posted here with permission of the IEEE. Such permission of the IEEE does not in any way imply IEEE endorsement of any of the University of Pennsylvania's products or services. Internal or personal use of this material is permitted. However, permission to reprint/republish this material for advertising or promotional purposes or for creating new collective works for resale or redistribution must be obtained from the IEEE by writing to pubs-permissions@ieee.org. By choosing to view this document, you agree to all provisions of the copyright laws protecting it.

This paper is posted at ScholarlyCommons. https://repository.upenn.edu/be_papers/136

For more information, please contact repository@pobox.upenn.edu. 


\title{
Self-organized cortical map formation by guiding connections
}

\begin{abstract}
We describe an algorithm for self-organizing connections from a source array to a target array of neurons that is inspired by neural growth cone guidance. Each source neuron projects a Gaussian pattern of connections to the target layer. Learning modifies the pattern center location. The small number of parameters required to specify connectivity has enabled this algorithm's implementation in a neuromorphic silicon system. We demonstrate that this algorithm can lead to topographic feature maps similar to those observed in the visual cortex, and characterize its operation as function maximization, which connects this approach with other models of cortical map formation.
\end{abstract}

\section{Keywords}

Gaussian distribution, neural nets, self-organising feature maps, function maximization, guiding connections, learning modified pattern center location, neural growth cone guidance, neuromorphic silicon systems, self-organized cortical map formation, source neuron Gaussian connection pattern, source/target array self-organizing connections, topographic feature maps, visual cortex

\section{Comments}

Copyright IEEE 2005. Reprinted from:

Lam, S.Y.M.; Shi, B.E.; Boahen, K.A., "Self-organized cortical map formation by guiding connections," Circuits and Systems, 2005. ISCAS 2005. IEEE International Symposium on, vol., no., pp. 5230-5233 Vol. 5, 23-26 May 2005

URL: http://proxy.library.upenn.edu:2994/stamp/stamp.jsp?arnumber=1465814\&isnumber=31469

This material is posted here with permission of the IEEE. Such permission of the IEEE does not in any way imply IEEE endorsement of any of the University of Pennsylvania's products or services. Internal or personal use of this material is permitted. However, permission to reprint/republish this material for advertising or promotional purposes or for creating new collective works for resale or redistribution must be obtained from the IEEE by writing to pubs-permissions@ieee.org. By choosing to view this document, you agree to all provisions of the copyright laws protecting it. 


\title{
SELF-ORGANIZED CORTICAL MAP FORMATION BY GUIDING CONNECTIONS
}

\author{
Stanley Y. M. Lam ${ }^{1}$, Bertram E. Shi ${ }^{1}$ and Kwabena A. Boahen ${ }^{2}$ \\ ${ }^{1}$ Dept. of EEE, HKUST, \{eelym,eebert\}@ust.hk and 20ept. of Bioengineering, UPenn, boahen@seas.upenn.edu
}

\begin{abstract}
We describe an algorithm for self-organizing connections from a source array to a target array of neurons that is inspired by neural growth cone guidance. Each source neuron projects a Gaussian pattern of connections to the target layer. Learning modifies the pattern center location. The small number of parameters required to specify connectivity has enabled this algorithm's implementation in a neuromorphic silicon system. We demonstrate that this algorithm can lead to topographic feature maps similar to those observed in the visual cortex, and characterize its operation as function maximization, which connects this approach with other models of cortical map formation.
\end{abstract}

\section{GROWTH CONES}

Neuromorphic vision systems are commonly based upon models of biological neural circuits. Currently, the circuits and processing in the retina are the best understood [1], which has enabled neuromorphic engineers to implement fairly realistic silicon models of retinal processing [2]. However, as we move towards higher levels of processing in the brain, our knowledge about the neural circuitry decreases dramatically.

Given this lack of knowledge, it might seem an impossible task for neuromorphic engineers to design silicon models of neural processing in areas beyond the retina. However, rather than depending on explicit knowledge about neural circuitry, we may be able to wire circuits modelling these areas by exploiting another biological mechanism: self organized development.

This paper describes an algorithm for this development that is inspired by neurite outgrowth. During development, neurites (axons and dendrites) grow out from a neuron to connect it with other neurons to form a network. Neurite growth is controlled by a growth cone at the tip of the neurite [3]. Diffusible chemicals whose release are activity dependent, such as brain derived neurotrophic factor (BDNF) [4], can act as tropic factors, which guide growth cones [5].

Growth cone inspired development has recently been implemented in a neuromorphic silicon system, which models the feedforward connections from a source array of neurons to a target 2D array [6]. In this system, source neurons connect with a set of target neurons centered around a location in the target array with weights that decrease with distance from the center location. Output activity in the target array leads to release of electronic charge that diffuses through the target array. The charge models a tropic factor, and the system updates center locations such that they move towards areas with high concentration. Experimental results demonstrate that the system can start with random initial connections and gradually evolve the connections over time to replicate the topography of the source array in the target array.

This paper has three goals. First, it demonstrates that this approach can lead to retinotopic maps that also exhibit other properties associated with the primary visual cortex, such as ocular dominance and orientation selectivity. Second, it establishes the stability of this map formation process. Finally, it relates this approach to previously proposed algorithms for map formation.

Section 2 of this paper introduces the learning algorithm. Section 3 shows via simulation that this algorithm can lead to ocular dominance and orientation maps similar to those observed in cortex. Section 4 analyzes the dynamics of learning, and shows that it evolves in such a way as to maximize an energy function. The existence of this energy function establishes stability as well as the connection between this algorithm and previously proposed algorithms.

\section{SELF-ORGANIZED GUIDANCE}

We assume that two-dimensional (2D) arrays of source neurons send out axons to connect with a 2D layer of target neurons. For concreteness, we will consider the example system of geniculocortical connections, and refer to the source neurons as lateral geniculate nucleus (LGN) neurons and the target neurons as cortical neurons. We will use different arrays of LGN source neurons to model different types of neurons, e.g. left eye/right eye, or ON/ OFF.

We index position in the LGN by Greek letters, e.g., $\alpha \in \mathbb{R}^{2}$, and position in the cortex by Roman letters, e.g. $x \in \mathbb{R}^{2}$. For simplicity, we will assume a single array of LGN neurons. Let $a_{\mathrm{LGN}}(\alpha)$ be the activity (spike rate) of an LGN neuron at $\alpha$. Each LGN neuron has an axon, which terminates in cortex with an arbor centered at location $c(\alpha) \in \mathbb{R}^{2}$. The connection strength between the LGN neuron at $\alpha$ and a cortical neuron at $x$ is determined by an arbor (weight) function $A(x-c(\alpha))$, that decays with the distance between the cortical neuron and the arbor center. In the following, we let $A$ be a circularly symmetric Gaussian with standard deviation $\sigma_{A}$. We assume that the postsynaptic activity of each cortical neuron is linear in the total presynaptic activity weighted by the connection strength:

$$
a_{\mathrm{COR}}(x)=\sum_{\beta} \mathrm{A}(x-c(\beta)) a_{\mathrm{LGN}}(\beta)
$$

Inspired by the ability of growth cones to follow neurotropin gradients and the activity dependent release of neurotropin, we model development by allowing the arbor center to move up its local neurotropin concentration gradient when it is active. The 
neurotropin concentration has both an activity dependent component and an activity independent component. For the activity dependent component, cortical neurons release neurotropin in an amount that is proportional to their post-synaptic activity with a constant of proportionality $T_{1}$. The neurotropin diffuses through cortex, which we model by convolving it with a circularly symmetric 2D Gaussian with standard deviation $\sigma_{D}$. Since the neurotropin concentration will be high where the activity is high, movement up the gradient will attract the arbor centers of coactive LGN neurons towards each other. For the activity independent component, we assume that there is an uptake (removal) that is proportional to the total afferent synaptic strength at each cortical location $x$ with constant of proportionality $T_{2}$. By reducing the neurotropin concentration in areas of high synaptic density, the uptake prevents the arbor centers from clustering too closely due to the attraction caused by the activity dependent component.

Mathematically, if $n(x)$ is the neurotropin concentration at cortical location $x$, then

$$
n(x)=T_{1} \sum_{\beta} G(x-c(\beta)) a_{\mathrm{LGN}}(\beta)-T_{2} \sum_{\alpha} A(x-c(\alpha))
$$

where $G(x)$ is a Gaussian with zero mean and variance $\sigma_{G}^{2}=\sigma_{D}^{2}+\sigma_{A}^{2}$, which reflects the net effect of the axonal arbor and neurotropin diffusion. For the purposes of computing the neurotropin concentration, we have assumed that the number of cortical neurons is much larger than the number of LGN neurons, so that the cortex can be approximated as a continuous sheet of neurons, where each location has an associated neuron. This simplifies the mathematical expressions by allowing us to combine the Gaussian describing the arbor and the Gaussian describing the diffusion into a single Gaussian. Assuming a discrete array would not change the model significantly.

If each axon center moves in the direction of the neurotropin gradient at a rate that is proportional to the afferent activity as well as the size of the gradient, then

$$
\frac{d c(\alpha)}{d t}=k \cdot a_{\mathrm{LGN}}(\alpha) \cdot \nabla n(c(\alpha))
$$

where $k$ is a constant of proportionality. Substituting (1) and assuming that the axon movement is slow compared to the rate at which the LGN activity changes so that the net motion is approximately proportional to the expected value of the gradient, we obtain

$$
\begin{aligned}
\frac{d c(\alpha)}{d t}= & k \cdot\left[T_{1} \sum_{\beta} \nabla G(c(\alpha)-c(\beta)) R_{\mathrm{LGN}}(\alpha, \beta)\right. \\
& \left.-T_{2} m_{\mathrm{LGN}}(\alpha) \sum_{\beta} \nabla A(c(\alpha)-c(\beta))\right]
\end{aligned}
$$

where $R_{\mathrm{LGN}}(\alpha, \beta)=E\left[a_{\mathrm{LGN}}(\alpha) a_{\mathrm{LGN}}(\beta)\right]$ is the correlation between the activity at LGN neurons $\alpha$ and $\beta$, and $m_{\mathrm{LGN}}(\alpha)=E\left[a_{\mathrm{LGN}}(\alpha)\right]$ is the mean activity of LGN neuron $\alpha$.

In most Hebbian models of development, an initial large population of connections is pruned to a smaller number of connections by modifying individual synaptic weights [7][8]. In this algorithm, the initial and final number of weights are equal. All weights from a source neuron are modified simultaneously through movement of the axon center.

\section{CORTICAL MAP FORMATION}

This section shows via computer simulation that the model can replicate retinotopic maps that exhibit ocular dominance columns and orientation columns similar to those observed in the cortex [9].

\subsection{Topographic Map Formation}

To illustrate topographical map formation, we simulated the connectivity from a 10 by 10 array of LGN neurons to cortex. We assumed two different tilings for the LGN array: rectangular and hexagonal. In both cases, the correlation between the activities of LGN cells was given by the same correlation function:

$$
R_{\mathrm{LGN}}(\alpha, \beta)=\left(2 \pi \sigma_{\text {in }}^{2}\right)^{-1} \exp \left(-(\alpha-\beta)^{2} /\left(2 \sigma_{\text {in }}^{2}\right)\right)
$$

The correlation between the activity of two LGN neurons decreases with distance.

We initialized the arbor centers near the origin of cortex according to a Gaussian distribution with standard deviation 0.5. Both tilings used the same initial conditions. The arbor center locations evolved according to (3) until convergence.

Figure 1 shows the final locations of the arbor centers, as well as snapshots of the evolution of the axon centers as they evolve. In both cases, the topography in the cortex replicates the original LGN topography up to a rotation. These results demonstrate that the algorithm can lead to self-organized topographical mappings, and that the process does not critically depend upon assumptions about the geometry of the topography in the source array.

\subsection{Ocular Dominance Map Formation}

To model ocular dominance map formation, we assumed two rectangular arrays of LGN neurons, modelling cells from the left and right eyes. Corresponding cells in the two arrays were assumed to serve the same location in visual space. All the cells terminate on the same layer in cortex. The activity of each LGN neuron was assumed to be correlated with activity in LGN neurons at nearby visual locations in both the same and the contralateral eye, but the correlation was weaker for activity in the contralateral eye. Mathematically, the correlation between the activity in LGN neurons corresponding to the same eye was given by (4). The correlation between LGN neurons in different eyes was equal to (4) multiplied by a constant $0 \leq k_{\mathrm{LR}} \leq 1$.

We simulated two 20 by 20 rectangular arrays of LGN neurons connecting to a 2D cortical plane sampled at a 200 by 200 array of points. We initialized the arbor centers on two noisy rectangular grids. Each grid was formed by taking a regular rectangular grid and perturbing each point by a independent random offset drawn from a circularly symmetric 2D Gaussian distribution whose standard deviation was equal to half the unperturbed distance between adjacent grid locations.

Figure 2 shows the resulting ocular dominance map, where cortical locations are labelled black or white depending upon whether 


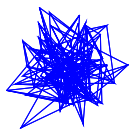 \\ $\mathrm{t}=0.0$}

$\mathrm{t}=0.1$

(a)
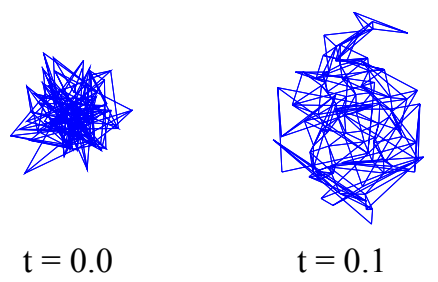

$\mathrm{t}=0.1$

(b)

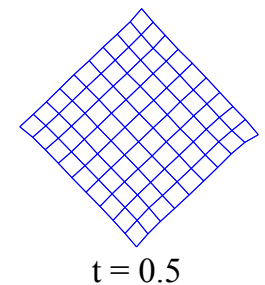

$\mathrm{t}=0.5$

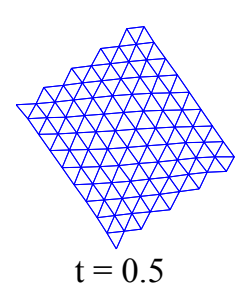

$\mathrm{t}=0.5$

Fig. 1: Topographic map formation. Grid points represent the locations of arbor centres of LGN cells in cortex. Neighboring LGN cells are connected by lines. Final spacing between nearest neighbors is approximately 0.3. (a) LGN cells arranged as a rectangular array. (b) LGN cells arranged as a hexagonal array. Parameters: $\sigma_{\text {in }}=2, \sigma_{A}=1, \quad \sigma_{G}=2.24, T_{1}=643.37$, $T_{2}=8.802$.

the total afferent strength from the left or right eye is greater. Similar to the ocular dominance maps observed in cortex, the map exhibits bands of ocular dominance with a characteristic width common to the left and right eyes.

\subsection{Orientation Map Formation}

To model the emergence of orientation columns, we assume two rectangular arrays of LGN neurons, representing ON center type cells and OFF center type cells. Corresponding points in the two array represent the same visual location. We assume the correlation between ON and OFF LGN cells has the form of a "Mexican hat":

$$
\begin{aligned}
& R_{\mathrm{LGN}}^{\mathrm{ON}-\mathrm{ON}}(\alpha, \beta)=R_{\mathrm{LGN}}^{\mathrm{OFF}-\mathrm{OFF}}(\alpha, \beta)=\left|R_{\mathrm{LGN}}(\alpha, \beta)\right|^{+} \\
& R_{\mathrm{LGN}}^{\mathrm{ON}-\mathrm{OFF}}(\alpha, \beta)=R_{\mathrm{LGN}}^{\mathrm{OFF-ON}}(\alpha, \beta)=\left|R_{\mathrm{LGN}}(\alpha, \beta)\right|^{-}
\end{aligned}
$$

where $|R|^{+}=\max \{R, 0\}$ and $|R|^{-}=\max \{-R, 0\}$. The expression $R_{\mathrm{LGN}}(\alpha, \beta)$ is the difference of two Gaussians with standard deviations $\sigma_{\text {in }}$ and $\sigma_{\text {surr }}$ representing the center and surround $\left(\sigma_{\text {in }}<\sigma_{\text {surr }}\right)$, where the surround Gaussian is weighted by a factor $k_{\mathrm{ON}-\mathrm{OFF}}$. The activity of a cell is positively correlated with activity of cells of the same type at close distances, and cells of opposite type at some distance away.

We simulated two 2D arrays of 20 by 20 LGN afferents terminating onto a 2D cortical plane sampled at a 200 by 200 array of points. As in the simulation of ocular dominance column formation, we initialized the axon locations with a noisy rectangular

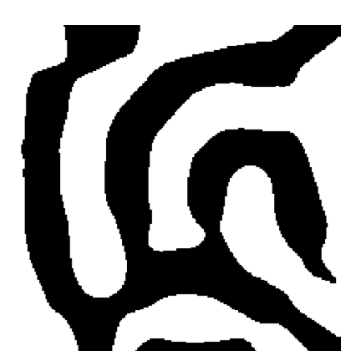

Fig. 2: Ocular dominance map. Cortical cells dominated by left and right eye input are shown in black and white respectively. Parameters: $\quad k_{\mathrm{LR}}=0.5, \quad \sigma_{\text {in }}=2 \sqrt{2}, \quad \sigma_{A}=1, \quad \sigma_{G}=\sqrt{5}$, $T_{1}=9562.37, T_{2}=82.72$.

topography, and allowed the locations to evolve until convergence.

Figure 3 shows the preferred orientation of the cortical neurons, where different colors indicate different preferred orientations. To determine each cell's preferred orientation, we presented sine wave gratings as the input to the LGN neurons. For each input orientation, we took the maximum over the responses of each cortical cell to gratings with different phases to obtain a phase invariant orientation response $O(\theta, x)$ where $\theta$ indicates the input orientation and $x$ indicates the cortical cell location. At each location, we combine the responses from different orientations into a complex vector,

$$
V_{R}(x)=\sum_{\theta} O(\theta, x) \cdot e^{j 2 \theta}
$$

whose phase angle determined the preferred orientation:

$$
\theta_{\text {pref }}(x)=0.5 \angle V_{R}(x) \text {. }
$$

\section{GRADIENT ASCENT LEARNING DYNAMICS}

In this section, we show that the equation describing the learning dynamics (3) can be expressed as gradient ascent on a function $E$.

Consider the following function of the afferent axon centers $\{c\}$ :

$$
\begin{aligned}
E(\{c\})= & \frac{T_{1}}{2} \sum_{\alpha, \beta} G(c(\alpha)-c(\beta)) R_{\mathrm{LGN}}(\alpha, \beta) \\
& -\frac{T_{2}}{2} \sum_{\alpha, \beta} m_{\mathrm{LGN}}(\alpha) A(c(\alpha)-c(\beta))
\end{aligned}
$$

Differentiating $E(\{c\})$ with respect to $c(\alpha)$, we find that the dynamics in our model can be written as:

$$
\frac{d c(\alpha)}{d t}=k \frac{d E(\{c\})}{d c(\alpha)}
$$

By formulating the learning dynamics as a process of gradient ascent, we immediately establish the stability of the map formation process. Since the function $E$ is bounded from above and the value of $E$ increases along trajectories of the learning process, the function $E$ is a Lyapunov function for the learning dynamics.

The function $E$ also establishes a link between this algorithm and a wide class of previously proposed algorithms for cortical map 


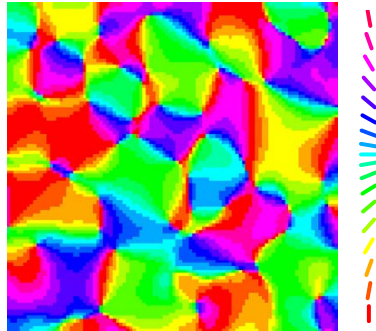

Fig. 3: Orientation map of cortical cells. The preferred orientation of neurons changes across the visual cortex. Different colors indicate different preferred orientations, according to the legend on the right hand side where the orientation of each colored bar indicates the preferred orientation of the cell. Parameters: $k_{\mathrm{ON}-\mathrm{OFF}}=2, \sigma_{\text {in }}=1, \sigma_{\text {surr }}=2, T_{1}=1647, T_{2}=35.21$.

formation, such as dimension reduction [10] and the elastic net [11]. Their dynamics can be expressed as the maximization of a $C$-measure [12]:

$$
C=\sum_{\alpha, \beta} G(c((\alpha), c(\beta))) \cdot F(\alpha, \beta)
$$

where $F$ is a measure of similarity applied to the input space and $G$ is a measure of similarity applied to the output space. Intuitively, this measure is maximized if similar points in the input space map to similar points in the output space.

The first term in (5) has the form of a $C$-measure, where the similarity measure in the output space is a Gaussian function of the Euclidean distance between the points whose variance depends on the axonal arbor and diffusion of neurotropin. The similarity measure in the input space is the correlation function that measure the degree of co-activity between the two points. One of the desirable properties of the $C$-measure we derive here is that the similarity measure in the cortical space initially decreases quadratically with the distance, but saturates at a zero lower bound for larger distances. This reduces the deleterious effect of outliers during map formation.

The $C$-measure we derive here arises naturally from the dynamics of the biologically inspired equations for axon guidance by neurotropin gradient following. In contrast, most algorithms for topographic map formation which can be formulated by a $C$-measure, derive this measure from "top-down" considerations of the attributes a "good" map should posses, such as coverage and continuity.

The final arbor center locations must balance the attraction introduced by the $C$-measure with repulsion introduced by the second term, which penalizes close distances between arbor centers. Thus, the ratio $T_{1} / T_{2}$ determines the final arbor center spacing in the topographic maps. The ocular dominance stripes form

because the $\mathrm{C}$-measure creates stronger attraction between neurons from the same eye because they are more strongly correlated. In the orientation map, the correlation function encourages $\mathrm{ON}$ neurons to cluster with OFF neurons slightly displaced in retinal position, leading to orientation tuned neurons.

\section{CONCLUSION}

We have described an algorithm for self organized map formation that is both amenable to silicon implementation and capable of replicating topographically organized maps similar to those observed in the visual cortex. We have also shown that the dynamics of the learning operate to maximize an objective function. These results indicate that in building neuromorphic systems for higher level processing, it may be possible to compensate for our lack of knowledge regarding the precise neural circuitry by exploiting self-organized development.

\section{ACKNOWLEDGEMENTS}

This work was supported by the US National Science Foundation BITS Program under grant EIA0130822 and by the Hong Kong Research Grants Council under grant HKUST6218/01E.

\section{REFERENCES}

[1] J. E. Dowling, The retina: an approachable part of the brain. Cambridge, Mass.: Belknap Press of Harvard University Press, 1987.

[2] K. A. Zaghloul and K. Boahen, "Optic Nerve Signals in a Neuromorphic Chip I: Outer and Inner Retina Models," IEEE Transactions on Biomedical Engineering, vol. 51, pp. 657-666, 2004.

[3] P. C. Letourneau, S. B. Kater, and E. R. Macagno (eds.), The Nerve Growth Cone, New York: Raven Press, 1991.

[4] A. K. McAllister, L. C. Katz, and D. C. Lo, "Neurotrophins and synaptic plasticity," Annual Reviews of Neuroscience, vol. 22, pp. 295-318, 1999.

[5] H. J. Song, G. L. Ming and M. M. Poo, "cAMP-induced switching in turning direction of nerve growth cones," Nature, vol. 288, pp. 275-279, 1997.

[6] B. Taba and K. Boahen, "Topographic Map Formation by Silicon Growth Cones," in Advances in Neural Information Processing Systems, vol. 15, S. Becker, S. Thrun, and K. Obermayer, Eds. MIT Press, 2003.

[7] R. Linsker, "From Basic Network Principles to Neural Architecture: Emergence of Spatial-Opponent Cells," Proc. Natl. Acad. Sci. USA, vol. 83, pp. 7508-7512, 1986.

[8] K. D. Miller, "A Model for the Development of Simple Cell Receptive Fields and the Ordered Arrangement of Orientation Columns through Activity-dependent Competition between ON- and OFF-Center Inputs," J. Neurosci, vol. 14, pp. 409-441, 1994.

[9] D. H. Hubel, Eye, brain and vision. New York: Scientific American Library, 1988.

[10] R. Durbin and G. Mitchison, "A dimension reduction framework for understanding cortical maps," Nature, vol. 343, pp. 644-647, 1990.

[11] G. J. Goodhill and D. J. Willshaw, "Application of the elastic net algorithm to the formation of ocular dominance stripes," Network, vol. 1, pp. 41-59, 1990.

[12] G. J. Goodhill and T. J. Sejnowski, "A unifying objective function for topographic mapping," Neural Computation, vol. 9, pp. 1291-1301, 1997. 